\title{
Low-Frequency Mechanical Stimulation Modulates Osteogenic Differentiation of C2C12 Cells
}

\author{
Ghazaleh Khayat, ${ }^{1}$ Derek H. Rosenzweig, ${ }^{1}$ Zohreh Khavandgar, ${ }^{2}$ Jingjing Li, ${ }^{2}$ \\ Monzur Murshed, ${ }^{2}$ and Thomas M. Quinn' ${ }^{1}$ \\ ${ }^{1}$ Soft Tissue Biophysics Laboratory, Department of Chemical Engineering, McGill University, 3610 University Street, \\ Montreal, QC, Canada H3A 0C5 \\ ${ }^{2}$ Faculty of Dentistry, McGill University, Montreal, QC, Canada H3A 0C5
}

Correspondence should be addressed to Thomas M. Quinn; thomas.quinn@mcgill.ca

Received 12 December 2012; Accepted 19 January 2013

Academic Editors: A. Chapel, C. Dong, S. M. Hwang, and B. Machalinski

Copyright ( $) 2013$ Ghazaleh Khayat et al. This is an open access article distributed under the Creative Commons Attribution License, which permits unrestricted use, distribution, and reproduction in any medium, provided the original work is properly cited.

\begin{abstract}
Mechanical stimulation influences stem cell differentiation and may therefore provide improved lineage specification control for clinical applications. Low-frequency oscillatory mechanical stimulation $(0.01 \mathrm{~Hz})$ has recently been shown to suppress adipogenic differentiation of mesenchymal stem cells, indicating that the range of effective stimulation frequencies is not limited to those associated with locomotion, circulation, and respiration. We hypothesized that low-frequency mechanical stimulation $(0.01 \mathrm{~Hz})$ can also promote osteogenic cell differentiation of myoblastic $\mathrm{C} 2 \mathrm{C} 12$ cells in combination with BMP-2. Results indicate that lowfrequency mechanical stimulation can significantly enhance osteogenic gene expression, provided that differentiation is initiated by a priming period involving BMP-2 alone. Subsequent application of low-frequency mechanical stimulation appears to act synergistically with continued BMP-2 exposure to promote osteogenic differentiation of C2C12 cells and can even partially compensate for the removal of BMP-2. These effects may be mediated by the ERK and Wnt signalling pathways. Osteogenic induction of $\mathrm{C} 2 \mathrm{C} 12$ cells by low-frequency mechanical stimulation is therefore critically dependent upon previous exposure to growth factors, and the timing of superimposed BMP-2 and mechanical stimuli can sensitively influence osteogenesis. These insights may provide a technically simple means for control of stem cell differentiation in cell-based therapies, particularly for the enhancement of differentiation toward desired lineages.
\end{abstract}

\section{Introduction}

Stem cell differentiation to bone is important to growth, development, and tissue maintenance; understanding of the cellular processes underlying osteogenesis is necessary for the development of cell-based therapies. Growth factor stimulation of stem cells is an initiating event which activates transcription factors that subsequently drive lineage specification. Bone-inducing agents [1] called bone morphogenetic proteins (BMPs) $[2,3]$ comprise a superfamily of growth factors that drive bone differentiation and adult bone maintenance. Among the BMP superfamily, BMP-2 is known to have a critical role in inducing osteogenic differentiation of mesenchymal stem cells [4]. Genes which are direct targets of BMP-2 include the transcription factors Runx2 and osterix, the matrix-associated proteins osteopontin and osteocalcin, and the osteogenic enzyme alkaline phosphatase [5]. It has also been established that the time course and duration of growth factor stimulation can have important effects on differentiation [6], and moreover BMP-2 efficacy is dose dependent [7].

In addition to the growth factor stimulation, mechanical stimulation can also affect osteogenic differentiation of stem cells $[8,9]$. Intracellular signalling pathways induced by mechanical stimulation in neurosensory and vascular cells can induce physiological responses which are much more rapid [10] than those induced by growth factors which can require minutes to hours [11]. Furthermore, downstream signalling events overlap between the two types of stimulation, potentially resulting in interactions between chemical and 
mechanical cues $[12,13]$. Mechanical stimulation utilizing amplitudes and frequencies consistent with the fundamental frequencies of locomotion $(0.1-1 \mathrm{~Hz})$ can induce osteogenic differentiation $[14,15]$. However, stimulation frequencies below this range have not been extensively studied in the context of osteogenesis, though they have been found effective in modulating differentiation of mesenchymal stem cells [16]. Since lower-frequency mechanical stimulation is often technically easier to apply, study of the effects of lowfrequency mechanical stimulation may create new avenues for integration of mechanical stimulation into protocols for stem cell differentiation in cell-based therapies.

Since mechanotransduction alone can enhance bone formation $[17,18]$ while BMP-2 is known to effectively drive osteogenesis, our goals were to explore the extent to which the complementary mechanisms interact during during osteogenic differentiation. Specifically, these stimuli were applied either together or in different sequences to elucidate their relative importance as osteogenesis progresses. The murine myoblastic $\mathrm{C} 2 \mathrm{C} 12$ cell line (of mesenchymal origin), which can differentiate into muscle, adipocyte, or osteoblastic lineages [3], was used. These cells have recently been shown to be sensitive to the sequence and timing of BMP-2 exposure during osteogenesis [19], making them an ideal candidate for testing interactions with mechanical stimulation as well.

\section{Materials and Methods}

2.1. Cell Culture. The cell line C2C12 (ATCC CRL-1772; American Type Culture Collection, Manassas, VA) was maintained on $10 \mathrm{~cm}$ diameter petri dishes in growth medium consisting of Dulbecco's modified Eagle medium (DMEM; Invitrogen, Burlington, ON) supplemented with $10 \%$ fetal bovine serum (FBS; Invitrogen) and $1 \%$ Penicillin-Streptomycin (Invitrogen) at $37^{\circ} \mathrm{C}$ in a humidified atmosphere of $5 \% \mathrm{CO}_{2}$ in air. Cells between passages 5 to 10 were used in all experiments. Cells were seeded on modified (as will be shown) high extension silicone rubber (HESR) culture dishes and maintained overnight for an attachment period. Seeding was performed using $5 \times 10^{5}$ cells on HESR dishes expanded to $20 \mathrm{~cm}^{2}$. After the 24 -hour attachment period, cells were typically $60-80 \%$ confluent and experiments were initiated.

\subsection{Preparation of Silicone Rubber Culture Surfaces. High} extension silicone rubber culture dishes (Cytomec $\mathrm{GmbH}$, Spiez, Switzerland) were modified to promote cell adhesion as previously described [16]. Briefly, unstretched HESR dishes were coated with $30 \%$ sulphuric acid for $15 \mathrm{~min}$ and then washed thoroughly with deionized water. Then surfaces were silanized with 1\% (3-aminopropyl) triethoxysilane for 2 hours at $70^{\circ} \mathrm{C}$ and washed thoroughly again. Surfaces were then functionalized with $5 \%$ (wt/vol) glutaraldehyed for another $15 \mathrm{~min}$ and washed. Next, surfaces were disinfected by rinsing with $70 \%$ ethanol, washed with phosphate buffered saline (PBS), mounted in a mechanical device (as will be shown), expanded to $20 \mathrm{~cm}^{2}$ culture surface area, and coated with protein by incubation with $50 \mu \mathrm{g} / \mathrm{mL}$ collagen type I in PBS overnight. On the next day, HESR surfaces were washed again with PBS prior to cell seeding.
2.3. Mechanical Stimulation. Mechanical stimulation was applied using an iris-like device (Cytomec) within which HESR culture dishes were mounted [16, 20] (Figures 1(a)$1(d))$. Approximately uniform expansion and contraction of the culture surface was applied by opening or closing the iris-like device. During mechanical stimulation, a continuous culture surface stretching and contraction was applied. Oscillatory stimulation occurred about a mean surface area of $20 \mathrm{~cm}^{2}$ with amplitude of $10 \%\left( \pm 2 \mathrm{~cm}^{2}\right)$ at a frequency of $0.01 \mathrm{~Hz}$. Unstimulated control cultures were maintained on HESR culture dishes mounted in the iris-like device and held to a fixed surface area of $20 \mathrm{~cm}^{2}$.

2.4. Experimental Protocols. As an initial control, the osteogenic potential of $\mathrm{C} 2 \mathrm{C} 12$ cells was tested under nonmechanically stimulated conditions during culture on tissue culture plastic in osteogenic medium (growth medium supplemented with $200 \mathrm{ng} / \mathrm{mL}$ BMP-2 (Invitrogen, Burlington, ON)). 200000 cells were seeded per well in a 6-well plate. After a 24-hour attachment period, cells were harvested from one well as a time zero control. Other wells were then induced with osteogenic medium for 6, 24, and 48 hours after which cells were collected for gene expression and histological analyses (as will be shown).

Four different experimental protocols (A, B, C, and D) were designed to investigate the combined effects of BMP2 induction and mechanical stimulation. In experiment $A$, cells were induced with BMP-2 for 48 hours while mechanical stimulation was only applied for the latter 24 hours. These were compared to controls under BMP-2 induction for 48 hours in the absence of any mechanical stimulation (Figure 3(a)). In experiment B, BMP-2 induction and mechanical stimulation were applied simultaneously for 48 hours and compared to controls where BMP-2 induction occurred in the absence of any mechanical stimulation (Figure 4(a)). Protocols A and B were designed to investigate how mechanical stimulation could modulate the time course of BMP-2 induction. In experiment C, cells were primed with BMP-2 for 24 hours after which medium was replaced with fresh growth medium and mechanical stimulation was applied for the next 24 hours. These were compared to controls primed with BMP-2 for 24 hours after which medium was replaced with growth medium but no mechanical stimulation was applied (Figure 5(a)). Experiment D was similar to experiment $\mathrm{C}$ except that the control culture involved BMP-2 induction for 48 hours without any mechanical stimulation (Figure 6(a)). Protocols C and D addressed the question of whether mechanical stimulation could replace BMP-2 induction.

2.5. Reverse Transcription and Quantitative Real-Time PCR. Total RNA was isolated using TRIzol Reagent (Invitrogen) following the manufacturer's protocol. Reverse transcription was performed using $500 \mathrm{ng}$ of total RNA in $20 \mu \mathrm{L}$ volume per reaction using the qScript cDNA synthesis kit following the manufacturer's instructions (Quanta Biosciences, Gaithersburg, MD). cDNA was diluted $1: 10$ and $1 \mu \mathrm{L}$ of each cDNA sample was loaded per reaction (in duplicate) using PerfeCTa 


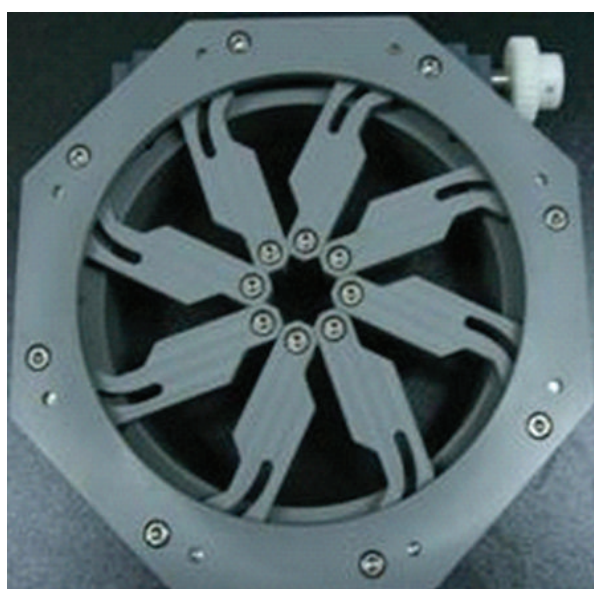

(a)

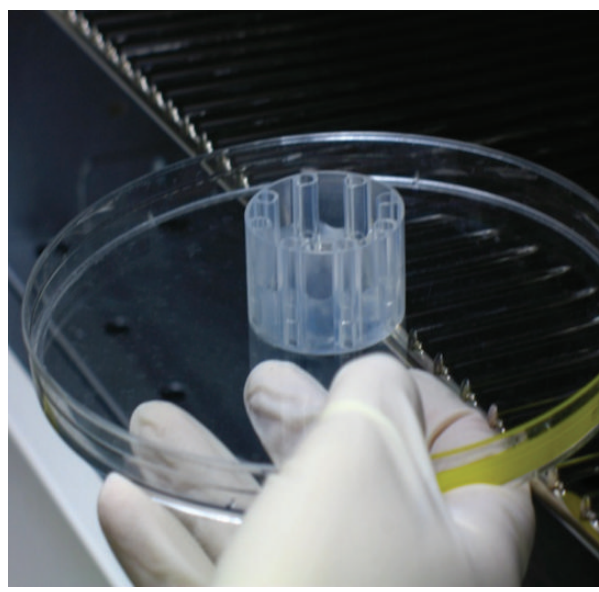

(c)

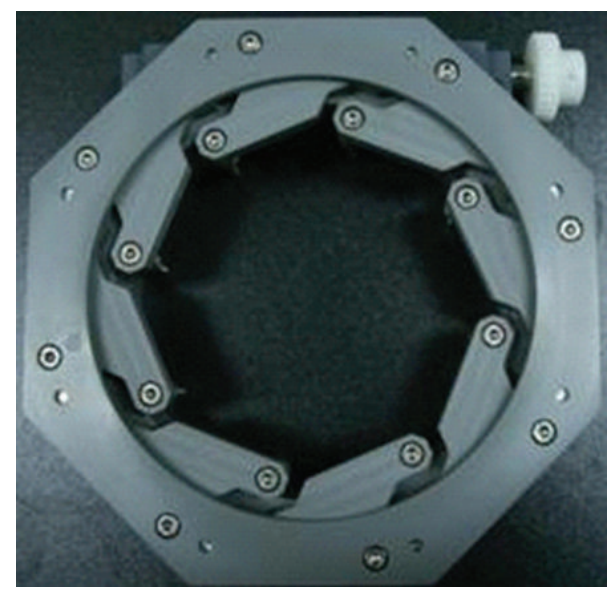

(b)

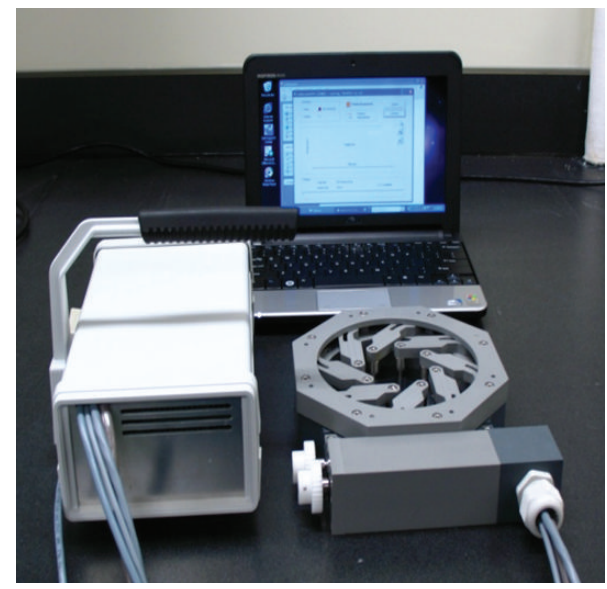

(d)

FIGURE 1: Experiments were conducted using an iris-like mechanical device which can stretch a high extension silicone rubber culture surface from 8 to $113 \mathrm{~cm}^{2}$. (a) Iris-like device in the fully closed position at $8 \mathrm{~cm}^{2}$. Scale bar represents $2 \mathrm{~cm}$. (b) Iris-like device in the fully open position at $113 \mathrm{~cm}^{2}$. (c) HESR petri dish prior to mounting in the iris-like device. Scale bar represents $1 \mathrm{~cm}$. (d) Mechanical stimulation device connected to the computer (modified from [16]).

SYBR Green FastMix (Quanta Biosciences). Standard quantitative real-time PCR protocols were performed using HPRT for normalization as previously described [16]. PCR primers for the transcription factors Runx2 and osterix (Osx) and for alkaline phosphatase (Alpl), Osteopontin, collagen type I (Colla1), bone Gla protein, iso-bone sialoprotein (Ibsp), osteoprotegerin (OPG), and HPRT [19] were generated exactly as described elsewhere [21].

2.6. Western Blotting. $\mathrm{C} 2 \mathrm{C} 12$ cells were lysed in lysis buffer (20 mM Tris (pH 7.4), $150 \mathrm{mM} \mathrm{NaCl}, 1 \mathrm{mM}$ EDTA, 0.5\% Triton X-100, and $1 \mathrm{mM} \beta$-glycerophosphate supplemented with complete EDTA-free protease inhibitor cocktail). $10 \mu \mathrm{g}$ of total protein was run on a $10 \%$ SDS-PAGE gel and transferred to nitrocellulose membranes. Membranes were blocked in 5\% BSA for 2 hours. For ERK1/2 analysis, blots were probed with antibodies against p42/p44 (1:2000, Cell Signalling), phospho-p42/p44 (1:1000, Cell Signalling), phospho-p38 (1:1000, Cell Signalling), and phospho-JNK (1:1000, Cell
Signalling) followed by anti-rabbit horseradish peroxidaseconjugated secondary antibody (1:10000, Cell Signalling). For Wnt signaling, blots were probed with antibodies against $\beta$-catenin $(1: 1000, \mathrm{Abcam})$ and $\alpha$-tubulin $(1: 1000, \mathrm{Abcam}$, Cambridge, MA), followed by incubation with anti-mouse horseradish peroxidase-conjugated secondary antibody (1:5000, Cell Signalling). Washed membranes were developed using Super Signal West Pico Substrate (Thermo Scientific, Rockford, IL) and BioMax MR film (Eastman Kodak).

2.7. Histology. $\mathrm{C} 2 \mathrm{C} 12$ cells were washed with deionized water and fixed with $4 \%$ paraformaldehyde (Alfa Aesar, Ward Hill, MA) for $2 \mathrm{~min}$. Formaldehyde was then removed by washing with PBS and ALP assay was immediately performed according to the manufacturer's instructions (Alkaline Phosphatase Detection Kit, Millipore). Images were captured using a Zeiss Axiovert 40C microscope equipped with a Canon Powershot A640 digital camera attached to a Zeiss MC80DX 1.0x tube adapter. 


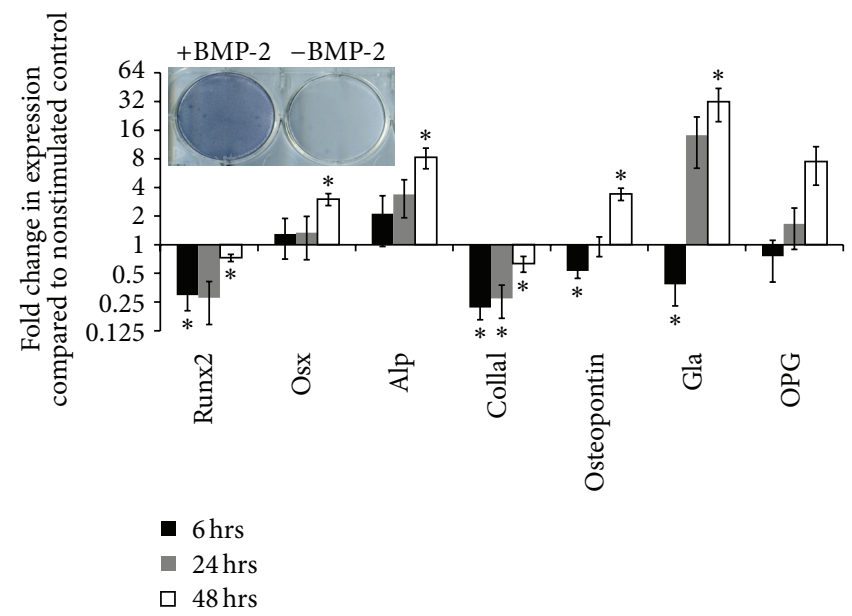

FIGURE 2: Osteogenic medium induces bone-like differentiation of C2C12 cells. RT-qPCR results for fold changes in Runx2, Osx, Alp, Collal, Osteopontin, bone Gla protein, and OPG expression after 6 hours (black bars), 24 hours (grey bars), and 48 hours (white bars), normalized to time zero controls (prior to exposure to osteogenic medium). Mean \pm SEM, $n=3 ;{ }^{*}$ represents a significant $(P<0.05)$ difference between BMP-2-treated and untreated cultures. Inset: cells treated with BMP-2 stained strongly positive for ALP activity (purple) after 48 hours. Scale bar represents $10 \mathrm{~mm}$.

2.8. Statistical Analysis. All experiments were performed at least three times. Statistical significance of differences between mechanically stimulated cultures and unstimulated controls was evaluated using two-tailed unequal variance $t$ tests $(P<0.05)$.

\section{Results}

3.1. Osteogenic Differentiation of C2C12 Cells in the Presence of BMP-2 Is Time Sensitive. C2C12 cells cultured in osteogenic medium for 6,24 , and 48 hours specifically began differentiating towards an osteoblast-like phenotype. qPCR analysis indicated significant changes in Runx2, Osx, Alp, Collal, Osteopontin, and bone Gla protein after 48 hours of induction with BMP-2 (Figure 2). While most genes were downregulated compared to time zero controls after 6 hours, there were trends for increases in all of them with time. Strong alkaline phosphatase activity was clearly visible (purple stain) after 48 hours in BMP-2-treated samples versus untreated $\mathrm{C} 2 \mathrm{C} 12$ cells (Figure 2 inset).

3.2. Low-Frequency Stimulation Enhances Osteogenesis in C2C12 Cells after BMP-2 Priming. Experiment A included 24 hours of priming with BMP-2 followed by 24 hours of superimposed mechanical stimulation (Figure 3(a)). This protocol had no obvious effect on gross morphology (Figure 3(b)). As indicated by qPCR (Figure 3(c)), low-frequency mechanical stimulation significantly upregulated expression of Runx2, Osx, Alp and Collal with respect to BMP-2-induced controls at 48 hours. Upregulation of osteopontin also approached significance $(P=0.054)$. Mechanical stimulation caused trends for upregulation of bone Gla protein and OPG versus controls but these were not statistically significant (Figure 3(c)).

3.3. Low-Frequency Stimulation without BMP-2 Priming Does Not Enhance Osteogenesis. In experiment B, BMP-2 induction and low-frequency mechanical stimulation were applied simultaneously for 48 hours (Figure 4(a)). qPCR analysis demonstrated no significant changes in osteogenic gene expression compared to nonmechanically stimulated controls at 48 hours (Figure 4(b)), although Runx2 appeared slightly upregulated $(P=0.08)$. No changes in cell morphology were observed (not shown).

3.4. Low-Frequency Stimulation Is Sufficient to Modulate Osteogenesis after BMP-2 Priming. In experiment C, cells were primed for 24 hours with BMP-2, after which low-frequency mechanical stimulation was applied for 24 hours in the absence of BMP-2 and compared to controls without BMP-2 (Figure 5(a)). qPCR results indicated significant upregulation of Collal and strong trends for upregulation of Osx and Alp which approached significance $(P=0.07$ and 0.08$)$ versus nonmechanically stimulated controls (Figure 5(b)). In contrast, osteopontin was significantly downregulated versus controls.

3.5. BMP-2-Induced Osteogenesis Masks the Effects of LowFrequency Mechanical Stimulation. In experiment D, mechanically stimulated cells from experiment $\mathrm{C}$ were compared to controls in which BMP-2 was present for 48 hours (Figure 6(a)). Less osteogenic differentiation was observed in cells exposed to low-frequency mechanical stimulation versus those induced with BMP-2 alone for 48 hours (Figure 6(b)). These effects were particularly significant for Runx2, Osx and Alp.

3.6. Low-Frequency Mechanical Stimulation Alters MAPK and Wnt Signaling in $\mathrm{C} 2 \mathrm{C} 12$ Cells. In experiment A, Western blot analysis of protein lysates at 48 hours showed that priming with BMP-2 followed by superposition of low-frequency mechanical stimulation downregulated ERK and p38 activity (Figure 7). In experiment B, Western blot analysis revealed that concomitant application of low-frequency mechanical 


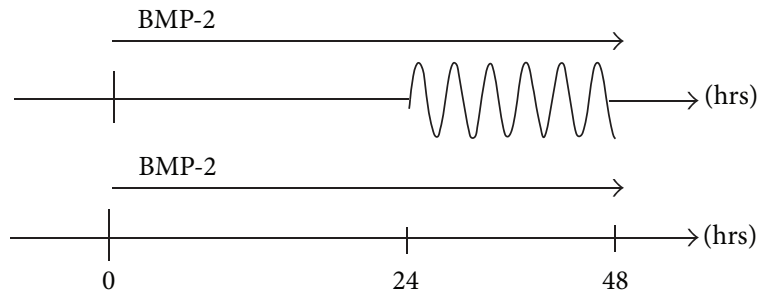

(a)

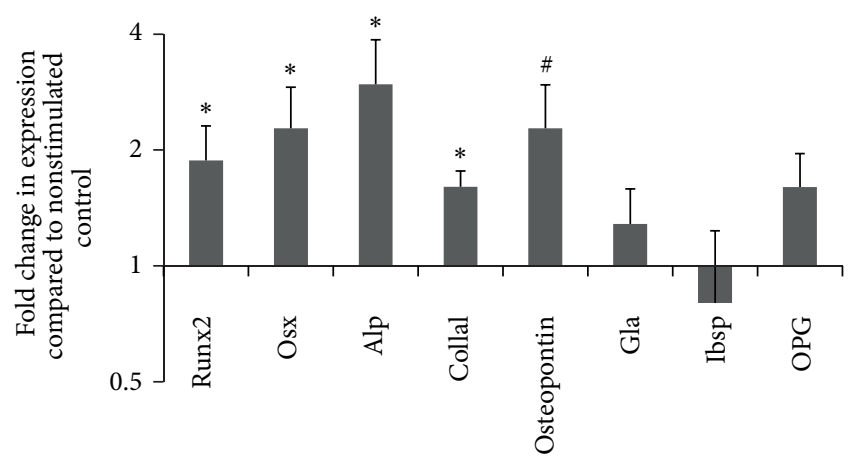

(b)
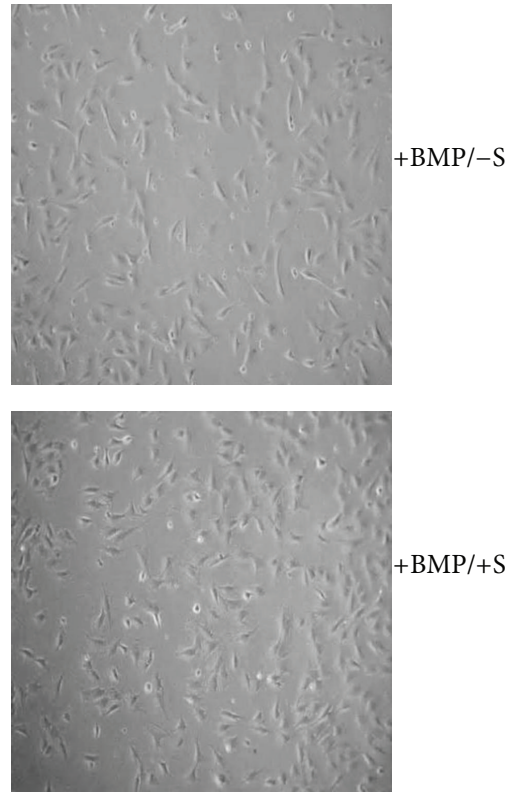

(c)

FIGURE 3: (a) Experimental protocol A involved BMP-2 induction for 48 hours with mechanical stimulation only for the latter 24 hours. Controls were BMP-2-induced for 48 hours in the absence of any mechanical stimulation. (b) Mechanical stimulation in the presence of BMP-2 enhanced osteogenic gene expression versus unstimulated controls. Mean \pm SEM, $n=6$; ${ }^{*}$ represents $P<0.05 ;{ }^{\#}$ represents $P=0.054$. (c) Morphology of C2C12 cells after 48 hours in experimental cultures (top) and controls (bottom). Scale bar represents $200 \mu \mathrm{m}$.

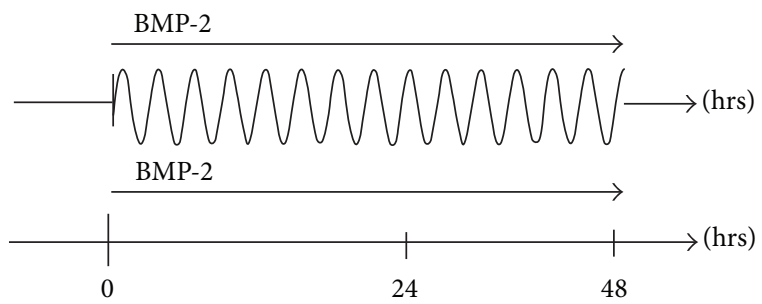

(a)

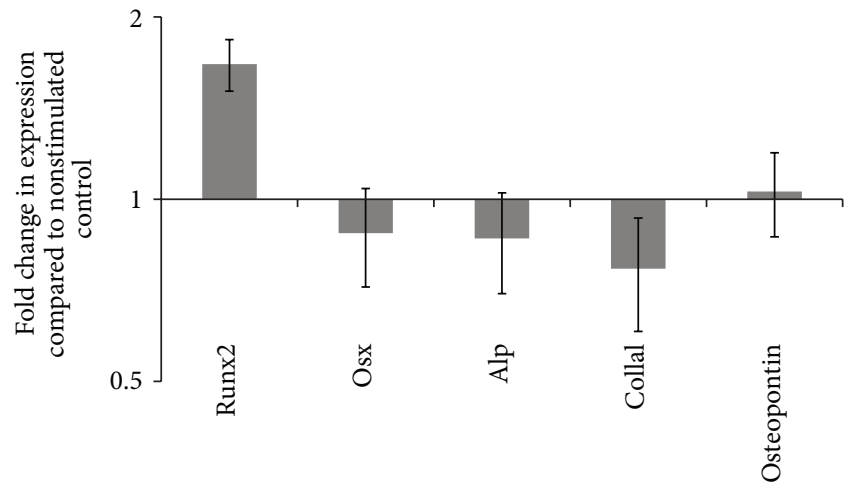

(b)

FIGURE 4: (a) Experimental protocol B involved 48 hours of simultaneous BMP-2 induction and mechanical stimulation compared to controls with BMP-2 induction only. (b) Fold differences in osteogenic gene expression at 48 hours. Mean \pm SEM, $n=3$.

stimulation and BMP-2 without priming caused an increase in ERK activity. For experiment $C$, no changes were observed in MAPK activity between cells primed with BMP-2 and then mechanically stimulated without BMP-2 versus nonmechanically stimulated controls which were primed and then cultured without BMP-2. In experiment D, when mechanical stimulation after priming was compared to 48 hours of BMP2-induced controls, an increase in ERK activity was observed with no detectable change in p38 activity (not shown). JNK activity was below detection for all experiments (not shown). When analyzing Wnt signalling, no detectable changes in $\beta$-catenin were observed under most experimental conditions, with the exception of a slight increase in experiment D (Figure 7).

\section{Discussion}

Previous studies have shown that BMP-2 induces osteogenesis through upregulation of the key transcription factors Runx2 [22] and Osx (Osterix) [23, 24]. The present results confirm these findings and moreover demonstrate that lowfrequency mechanical stimulation can enhance osteogenic 


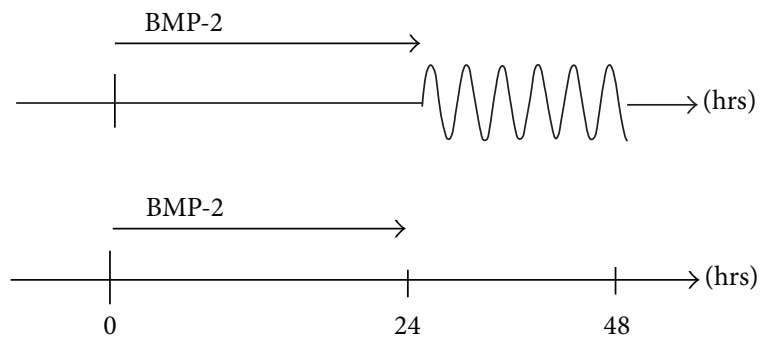

(a)

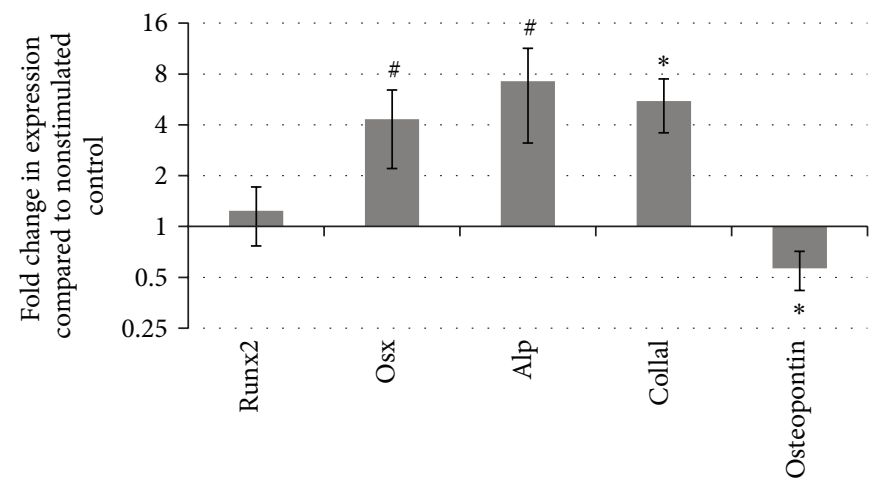

(b)

Figure 5: (a) Protocol C involved 24-hour priming with BMP-2 followed by changing of medium and mechanical stimulation for next 24 hours. Controls were identically treated except for mechanical stimulation. (b) Fold differences in osteogenic gene expression at 48 hours. Mean \pm SEM, $n=5$; ${ }^{*}$ represents $P<0.05 ;{ }^{\#}$ represents $P<0.08$.

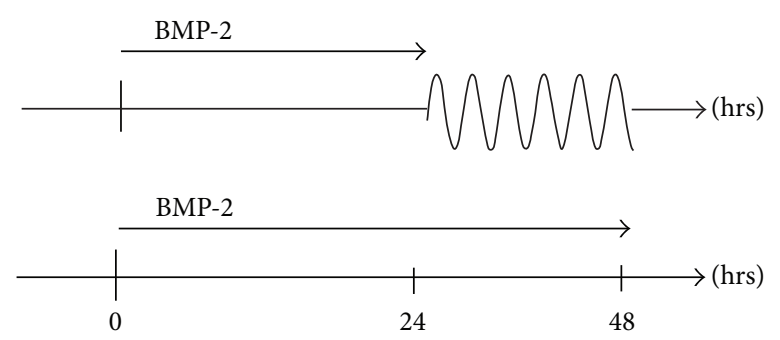

(a)

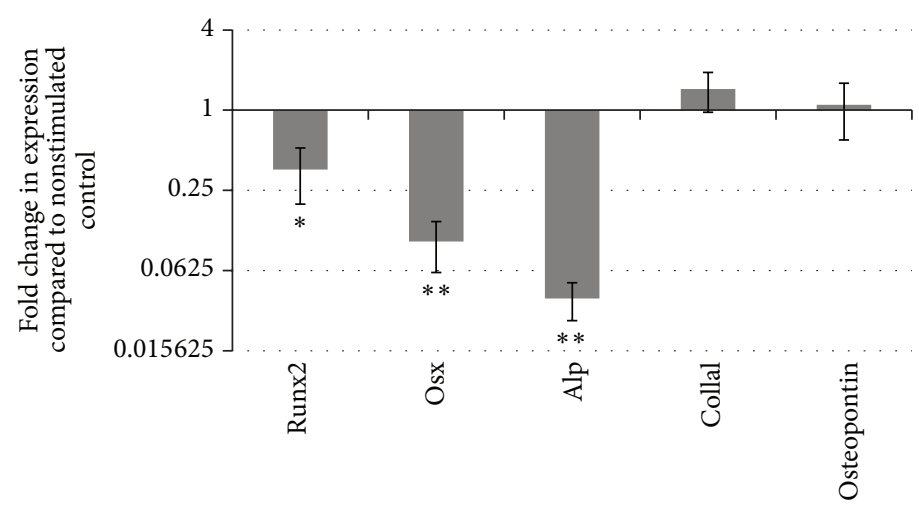

(b)

Figure 6: (a) Protocol D involved 24-hour priming with BMP-2 followed by changing of medium and mechanical stimulation for the next 24 hours. Controls were BMP-2-induced for 48 hours in the absence of any mechanical stimulation. (b) Fold differences in osteogenic gene expression at 48 hours. Mean $\pm \mathrm{SEM}, n=3$; ${ }^{*}$ represents $P<0.05$; ${ }^{* *}$ represents $P<0.01$.

differentiation of $\mathrm{C} 2 \mathrm{C} 12$ cells in the presence of BMP-2. BMP2 alone induces expression of bone marker genes such as alkaline phosphatase (Alp), Osteopontin, and collagen type I [25], and we have clearly shown here that concomitant low-frequency mechanical stimulation has a synergistic effect on $\mathrm{C} 2 \mathrm{C} 12$ osteogenic differentiation depending on the timing of these costimuli. These findings are consistent with those of previous studies involving significantly different mechanical conditions, notably higher oscillatory stimulation frequencies [26-28]. These previous studies employed frequencies between 0.1 and $1 \mathrm{~Hz}$ to show that mechanical stimulation can inhibit or induce stem cell differentiation to desired lineages [29-33]. The present results show that this upregulation in bone marker genes can also occur at frequencies as low as $0.01 \mathrm{~Hz}$, which are much lower than fundamental frequencies of locomotion. This indicates that enhancement in osteogenic differentiation of $\mathrm{C} 2 \mathrm{C} 12$ cells by oscillatory mechanical stimulation can occur for a wider range of frequencies than previously believed, similar to previous findings for inhibition of adipogenic differentiation [16].

It is known that BMP-2 can induce osteogenic differentiation of stem cells [34-36]. Moreover, exposure to BMP-2 is necessary for initiation of osteogenic differentiation of $\mathrm{C} 2 \mathrm{C} 12$ cells [19]. Our pilot studies established that low-frequency mechanical stimulation in the absence of BMP-2 exposure did not significantly drive osteogenic differentiation of $\mathrm{C} 2 \mathrm{C} 12$ cells by itself (data not shown). Therefore all experiments performed in the present study involved a 24-48-hour exposure to BMP-2 as a "priming" step to provide an environment within which mechanical effects on osteogenesis could be investigated. Interestingly, when mechanical stimulation was started at the same time as BMP-2 induction such that no priming period with BMP-2 alone occurred, no significant changes in osteogenic gene expression were observed (Figure 4). In contrast, synergistic effects on osteogenesis were observed when concomitant BMP-2 and mechanical stimuli were applied following priming by BMP-2 alone 


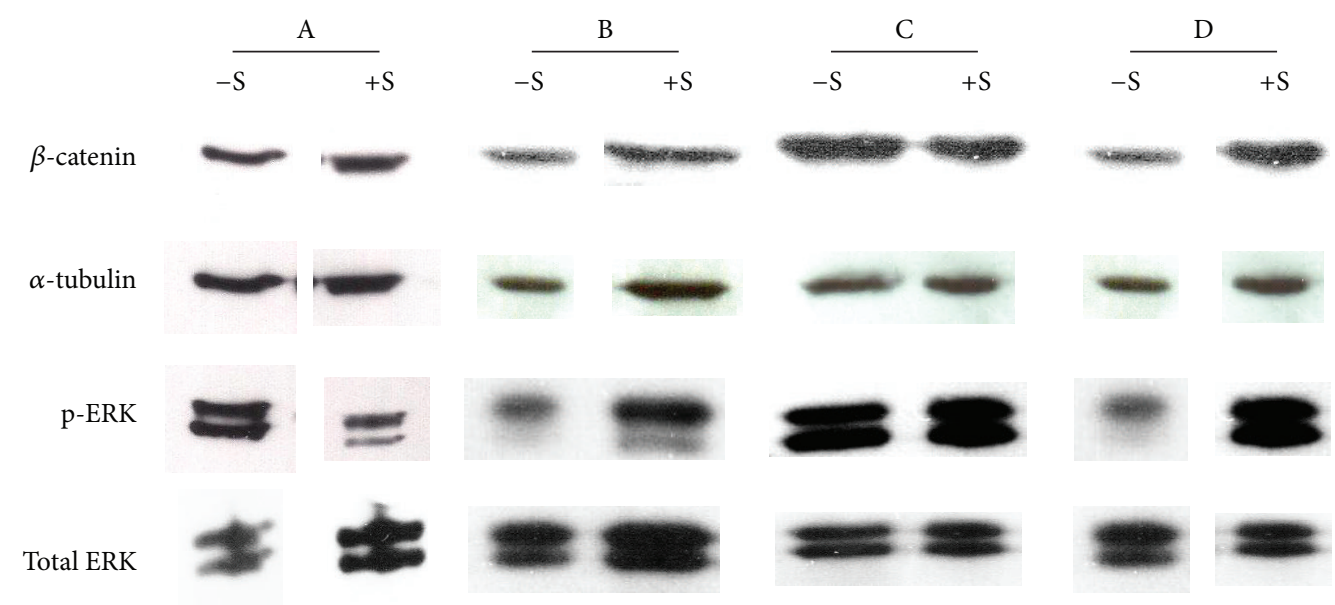

FIGURE 7: Western blot analysis of the effects of low-frequency mechanical stimulation on Wnt and MAPK signalling in C2C12 cells undergoing BMP-2-induced osteogenesis. Scanned images are representative of 4 independent experiments.

(Figure 3). Specifically, the early osteogenic markers Runx2, Osx, and Alp [37] and the late osteogenic marker Collal [38] were significantly upregulated versus nonmechanically stimulated controls. However, no significant changes in the other osteogenic markers Gla, Ibsp, or OPG were observed. Evidently, osteogenic effects of low-frequency mechanical stimulation on $\mathrm{C} 2 \mathrm{C} 12$ cells can be critically dependent upon previous exposure to growth factor stimulation, and the timing and sequence of superimposed BMP-2 and mechanical stimuli can sensitively influence osteogenesis.

After initiation of osteogenesis with BMP-2 priming, our latter experiments tested if mechanical stimulation in the absence of BMP-2 could further modulate osteogenesis or match the efficacy of continued BMP-2 exposure. Following 24 hours of BMP-2 priming, low-frequency mechanical stimulation significantly upregulated expression of Osx, Alp, and Collal versus controls where BMP-2 exposure stopped after priming (Figure 5). Conversely, osteopontin was slightly downregulated, but overall the effects of mechanical stimulation alone following BMP-2 priming indicated enhancement of osteogenesis. However, low-frequency mechanical stimulation after priming in the absence of BMP-2 could not match the efficacy of continued BMP-2 exposure in non-mechanically stimulated controls (Figure 6). Specifically, significantly diminished expression of Runx2, Osx, and Alp was observed in mechanically stimulated cells in competition with BMP-2-stimulated controls, though Collal and osteopontin were expressed at similar levels in both conditions. Therefore, it appears that low-frequency mechanical stimulation can enhance osteogenesis of C2C12 cells when BMP-2 concentration decreases; however, this enhancement is not as potent as continued high concentrations of BMP-2 $[19,26]$.

Recent studies utilizing high-frequency mechanical stimulation have indicated a role for mitogen-activated protein (MAP) kinase signaling in stem cell differentiation [39]. Other in vitro studies also implied that $\beta$-catenin is required downstream of BMP-2 for osteoblast mineralization [40]. The extracellular-related kinase (ERK) and p38 kinase pathways are strong candidates for this activity since previous work has shown that mechanical stimulation induces a rapid and transient change in both kinase activities in $\mathrm{C} 2 \mathrm{C} 12$ and osteoblast-like cells [41-43]. Also, the Wnt signalling pathway is involved in the response of bone-like cells to mechanical loading [44] and is also active during osteogenic differentiation of C2C12 cells [45-47]. Consistent with these findings, our Western blot data suggest that low-frequency mechanical stimulation following BMP-2 priming may act primarily through ERK and $\beta$-catenin (Wnt) signalling. However, we were unable to detect differences in p38 and JNK activities under our experimental conditions. Further studies are required to elucidate the differences between modes of mechanical stimulation in terms of the specific intercellular signaling pathways activated during osteogenic differentiation.

Perhaps the most obvious context within which varying growth factor concentrations and low-frequency mechanical stimulation act in concert on stem cells is during development $[48,49]$. It is perhaps unwarranted to extrapolate the findings of this in vitro study to more complex processes; however the present results suggest a model for interpretation of how these stimuli can contribute in a coordinated fashion during development. For example, during limb bud development, transient growth factor concentration gradients initiate stem cell differentiation and establish basic morphology [50], while subsequent low-frequency movement and growth may refine these patterns by modulating cytokine, growth factor, and ECM protein production, thus ultimately promoting terminal differentiation and structure [49]. These insights may enable future application of present findings for improved control of stem cell differentiation in cell-based therapies.

\section{Conflict of Interests}

One of the authors (T. M. Quinn) is a shareholder in the company Cytomec $\mathrm{GmbH}$, which makes the iris-like devices 
for cell culture on high extension surfaces used in this study. There is no further conflict of interests.

\section{Acknowledgments}

This paper was supported by the Canada Research Chairs and NSERC Discovery Grants Program.

\section{References}

[1] M. R. Urist, "Bone: formation by autoinduction," Science, vol. 150, no. 3698, pp. 893-899, 1965.

[2] D. D. Wang, R. Zeng, M. E. Yang et al., "Enhancement of bone formation by recombinant human bone morphogenetic protein-2/chitosan bone biomaterials," Journal of Clinical Rehabilitative Tissue Engineering Research, vol. 15, no. 25, pp. 45834586, 2011.

[3] M. R. Urist and B. S. Strates, "Bone morphogenetic protein," Journal of Dental Research, vol. 50, no. 6, pp. 1392-1406, 1971.

[4] H. M. Ryoo, M. H. Lee, and Y. J. Kim, "Critical molecular switches involved in BMP-2-induced osteogenic differentiation of mesenchymal cells," Gene, vol. 366, no. 1, pp. 51-57, 2006.

[5] C. A. Gersbach, R. E. Guldberg, and A. J. García, "In vitro and in vivo osteoblastic differentiation of BMP-2- and Runx2-engineered skeletal myoblasts," Journal of Cellular Biochemistry, vol. 100, no. 5, pp. 1324-1336, 2007.

[6] B. Shen, A. Wei, S. Whittaker et al., "The role of BMP-7 in chondrogenic and osteogenic differentiation of human bone marrow multipotent mesenchymal stromal cells in vitro," Journal of Cellular Biochemistry, vol. 109, no. 2, pp. 406-416, 2010.

[7] T. Katagiri, A. Yamaguchi, M. Komaki et al., "Bone morphogenetic protein- 2 converts the differentiation pathway of $\mathrm{C} 2 \mathrm{C} 12$ myoblasts into the osteoblast lineage," Journal of Cell Biology, vol. 127, no. 6 I, pp. 1755-1766, 1994.

[8] N. Kobayashi, T. Yasu, H. Ueba et al., "Mechanical stress promotes the expression of smooth muscle-like properties in marrow stromal cells," Experimental Hematology, vol. 32, no. 12, pp. 1238-1245, 2004.

[9] C. Zhang, J. Li, L. Zhang et al., "Effects of mechanical vibration on proliferation and osteogenic differentiation of human periodontal ligament stem cells," Archives of Oral Biology, vol. 57, no. 10, pp. 1395-1407, 2012.

[10] H. Sackin, "Stretch-activated ion channels," Kidney International, vol. 48, no. 4, pp. 1134-1147, 1995.

[11] S. M. Jones and A. Kazlauskas, "Connecting signaling and cell cycle progression in growth factor-stimulated cells," Oncogene, vol. 19, no. 49, pp. 5558-5567, 2000.

[12] S. Gallea, F. Lallemand, A. Atfi et al., "Activation of mitogenactivated protein kinase cascades is involved in regulation of bone morphogenetic protein-2-induced osteoblast differentiation in pluripotent C2C12 cells," Bone, vol. 28, no. 5, pp. 491-498, 2001.

[13] B. Sen, Z. Xie, N. Case, M. Ma, C. Rubin, and J. Rubin, "Mechanical strain inhibits adipogenesis in mesenchymal stem cells by stimulating a durable $\beta$-catenin signal," Endocrinology, vol. 149, no. 12, pp. 6065-6075, 2008.

[14] P. Zhang, Y. Wu, Z. Jiang, L. Jiang, and B. Fang, "Osteogenic response of mesenchymal stem cells to continuous mechanical strain is dependent on ERK1/2-Runx2 signaling," International Journal of Molecular Medicine, vol. 29, pp. 1083-1089, 2012.
[15] J. Kopf, A. Petersen, G. N. Duda, and P. Knaus, "BMP2 and mechanical loading cooperatively regulate immediate early signalling events in the BMP pathway," BMC Biology, vol. 10, article 37, 2012.

[16] G. Khayat, D. H. Rosenzweig, and T. M. Quinn, "Low frequency mechanical stimulation inhibits adipogenic differentiation of C3H10T1/2 mesenchymal stem cells," Differentiation, vol. 83, pp. 179-184, 2012.

[17] E. Lau, S. Al-Dujaili, A. Guenther, D. Liu, L. Wang, and L. You, "Effect of low-magnitude, high-frequency vibration on osteocytes in the regulation of osteoclasts," Bone, vol. 46, no. 6, pp. 1508-1515, 2010.

[18] C. M. A. Reijnders, N. Bravenboer, P. J. Holzmann, F. Bhoelan, M. A. Blankenstein, and P. Lips, "In vivo mechanical loading modulates insulin-like growth factor binding protein-2 gene expression in rat osteocytes," Calcified Tissue International, vol. 80, no. 2, pp. 137-143, 2007.

[19] J. Li, Z. Khavandgar, S. H. Lin, and M. Murshed, "Lithium chloride attenuates BMP-2 signaling and inhibits osteogenic differentiation through a novel WNT/GSK3-independent mechanism," Bone, vol. 48, no. 2, pp. 321-331, 2011.

[20] H. Majd, P. J. Wipff, L. Buscemi et al., "A novel method of dynamic culture surface expansion improves mesenchymal stem cell proliferation and phenotype," Stem Cells, vol. 27, no. 1, pp. 200-209, 2009.

[21] H. P. Makarenkova, K. N. Gonzalez, W. B. Kiosses, and R. Meech, "Barx2 controls myoblast fusion and promotes MyoDmediated activation of the smooth muscle $\alpha$-actin gene," The Journal of Biological Chemistry, vol. 284, no. 22, pp. 1486614874, 2009.

[22] T. Komori, H. Yagi, S. Nomura et al., "Targeted disruption of Cbfal results in a complete lack of bone formation owing to maturational arrest of osteoblasts," Cell, vol. 89, no. 5, pp. 755764, 1997.

[23] C. Niger, F. Lima, D. J. Yoo et al., “The transcriptional activity of osterix requires the recruitment of Spl to the osteocalcin proximal promoter," Bone, vol. 49, pp. 683-692, 2011.

[24] A. Ulsamer, M. J. Ortuño, S. Ruiz et al., "BMP-2 induces osterix expression through up-regulation of Dlx 5 and its phosphorylation by p38," The Journal of Biological Chemistry, vol. 283, no. 7, pp. 3816-3826, 2008.

[25] O. A. Arosarena, F. E. del Carpio-Cano, R. A. dela Cadena, M. C. Rico, E. Nwodim, and F. F. Safadi, "Comparison of bone morphogenetic protein-2 and osteoactivin for mesenchymal cell differentiation: effects of bolus and continuous administration," Journal of Cellular Physiology, vol. 226, pp. 2943-2952, 2011.

[26] I. S. Kim, Y. M. Song, T. H. Cho, J. Y. Kim, F. E. Weber, and S. J. Hwang, "Synergistic action of static stretching and BMP-2 stimulation in the osteoblast differentiation of $\mathrm{C} 2 \mathrm{C} 12$ myoblasts," Journal of Biomechanics, vol. 42, no. 16, pp. 27212727, 2009.

[27] M. Iwata, S. Suzuki, K. Hayakawa, T. Inoue, and K. Naruse, "Uniaxial cyclic stretch increases glucose uptake into C2C12 myotubes through a signaling pathway independent of insulinlike growth factor I," Hormone and Metabolic Research, vol. 41, no. 1, pp. 16-22, 2009.

[28] S. H. Hook, H. J. Lee, W. T. Chung et al., "Cyclic mechanical stretch stimulates the proliferation of $\mathrm{C} 2 \mathrm{C} 12$ myoblasts and inhibits their differentiation via prolonged activation of $\mathrm{p} 38$ MAPK," Molecules and Cells, vol. 25, no. 4, pp. 479-486, 2008.

[29] Y. Shi, H. Li, X. Zhang et al., "Continuous cyclic mechanical tension inhibited Runx2 expression in mesenchymal stem cells 
through RhoA-ERK1/2 pathway," Journal of Cellular Physiology, vol. 226, no. 8, pp. 2159-2169, 2011.

[30] R. C. Solem, B. F. Eames, M. Tokita, and R. A. Schneider, "Mesenchymal and mechanical mechanisms of secondary cartilage induction," Developmental Biology, vol. 356, no. 1, pp. 28-39, 2011.

[31] A. Grossi, R. Lametsch, A. H. Karlsson, and M. A. Lawson, "Mechanical stimuli on C2C12 myoblasts affect myoblast differentiation, focal adhesion kinase phosphorylation and galectin-1 expression: a proteomic approach," Cell Biology International, vol. 35, no. 6, pp. 579-586, 2011.

[32] Y. F. Rui, P. P. Y. Lui, M. Ni, L. S. Chan, Y. W. Lee, and K. M. Chan, "Mechanical loading increased BMP-2 expression which promoted osteogenic differentiation of tendon-derived stem cells," Journal of Orthopaedic Research, vol. 29, no. 3, pp. 390396, 2011.

[33] C. E. Sarraf, W. R. Otto, and M. Eastwood, "In vitro mesenchymal stem cell differentiation after mechanical stimulation," Cell Proliferation, vol. 44, no. 1, pp. 99-108, 2011.

[34] T. Katagiri, A. Yamaguchi, T. Ikeda et al., "The non-osteogenic mouse pluripotent cell line, C3H10T1/2, is induced to differentiate into osteoblastic cells by recombinant human bone morphogenetic protein-2," Biochemical and Biophysical Research Communications, vol. 172, no. 1, pp. 295-299, 1990.

[35] A. Yamaguchi, T. Katagiri, T. Ikeda et al., "Recombinant human bone morphogenetic protein-2 stimulates osteoblastic maturation and inhibits myogenic differentiation in vitro," Journal of Cell Biology, vol. 113, no. 3, pp. 681-687, 1991.

[36] E. A. Wang, D. I. Israel, S. Kelly, and D. P. Luxenberg, "Bone morphogenetic protein-2 causes commitment and differentiation in C3H10T1/2 and 3T3 cells," Growth Factors, vol. 9, no. 1, pp. 57-71, 1993.

[37] E. Kaivosoja, S. Myllymaa, Y. Takakubo et al., "Osteogenesis of human mesenchymal stem cells on micro-patterned surfaces," Journal of Biomaterials Applications, 2011.

[38] M. Q. Hassan, R. S. Tare, H. L. Suk et al., "BMP2 commitment to the osteogenic lineage involves activation of Runx 2 by DLX3 and a homeodomain transcriptional network," The Journal of Biological Chemistry, vol. 281, no. 52, pp. 40515-40526, 2006.

[39] N. Nakai, F. Kawano, Y. Oke et al., "Mechanical stretch activates signaling events for protein translation initiation and elongation in C2C12 myoblasts," Molecules and Cells, vol. 30, no. 6, pp. 513$518,2010$.

[40] G. Rawadi, B. Vayssière, F. Dunn, R. Baron, and S. RomanRoman, "BMP-2 controls alkaline phosphatase expression and osteoblast mineralization by a Wnt autocrine loop," Journal of Bone and Mineral Research, vol. 18, no. 10, pp. 1842-1853, 2003.

[41] C. Rauch and P. T. Loughna, "Stretch-induced activation of ERK in myocytes is p38 and calcineurin-dependent," Cell Biochemistry and Function, vol. 26, no. 8, pp. 866-869, 2008.

[42] J. Rubin, C. Rubin, and C. R. Jacobs, "Molecular pathways mediating mechanical signaling in bone," Gene, vol. 367, no. 1-2, pp. 1-16, 2006.

[43] P. G. Ziros, A. P. R. Gil, T. Georgakopoulos et al., "The bonespecific transcriptional regulator Cbfal is a target of mechanical signals in osteoblastic cells," The Journal of Biological Chemistry, vol. 277, no. 26, pp. 23934-23941, 2002.

[44] J. A. Robinson, M. Chatterjee-Kishore, P. J. Yaworsky et al., "Wnt/ $\beta$-catenin signaling is a normal physiological response to mechanical loading in bone," The Journal of Biological Chemistry, vol. 281, no. 42, pp. 31720-31728, 2006.
[45] H. C. Yu, T. C. Wu, M. R. Chen, S. W. Liu, J. H. Chen, and K. M. C. Lin, "Mechanical stretching induces osteoprotegerin in differentiating $\mathrm{C} 2 \mathrm{C} 12$ precursor cells through noncanonical Wnt pathways," Journal of Bone and Mineral Research, vol. 25, no. 5, pp. 1128-1137, 2010.

[46] T. P. Hill, D. Später, M. M. Taketo, W. Birchmeier, and C. Hartmann, "Canonical Wnt/ $\beta$-catenin signaling prevents osteoblasts from differentiating into chondrocytes," Developmental Cell, vol. 8, no. 5, pp. 727-738, 2005.

[47] T. F. Day, X. Guo, L. Garrett-Beal, and Y. Yang, "Wnt/ $\beta$-catenin signaling in mesenchymal progenitors controls osteoblast and chondrocyte differentiation during vertebrate skeletogenesis," Developmental Cell, vol. 8, no. 5, pp. 739-750, 2005.

[48] O. Nilsson, E. A. Parker, A. Hegde, M. Chau, K. M. Barnes, and J. Baron, "Gradients in bone morphogenetic protein-related gene expression across the growth plate," Journal of Endocrinology, vol. 193, no. 1, pp. 75-84, 2007.

[49] J. H. Henderson and D. R. Carter, "Mechanical induction in limb morphogenesis: the role of growth-generated strains and pressures," Bone, vol. 31, no. 6, pp. 645-653, 2002.

[50] R. Zeller, J. López-Ríos, and A. Zuniga, "Vertebrate limb bud development: moving towards integrative analysis of organogenesis," Nature Reviews Genetics, vol. 10, no. 12, pp. 845-858, 2009. 


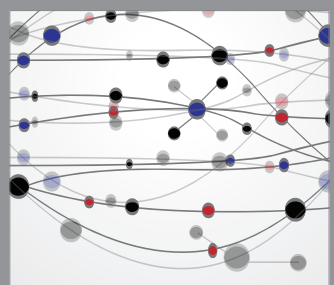

The Scientific World Journal
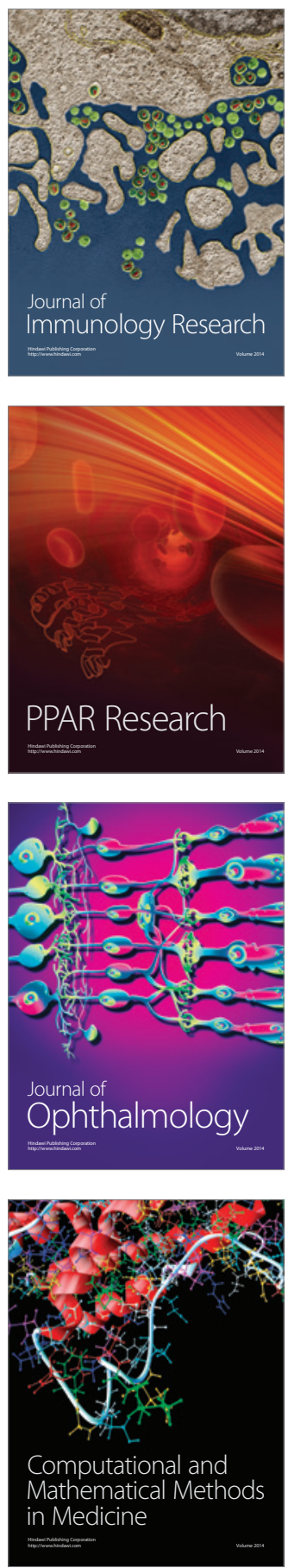

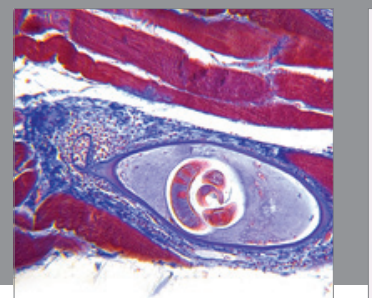

Gastroenterology

Research and Practice
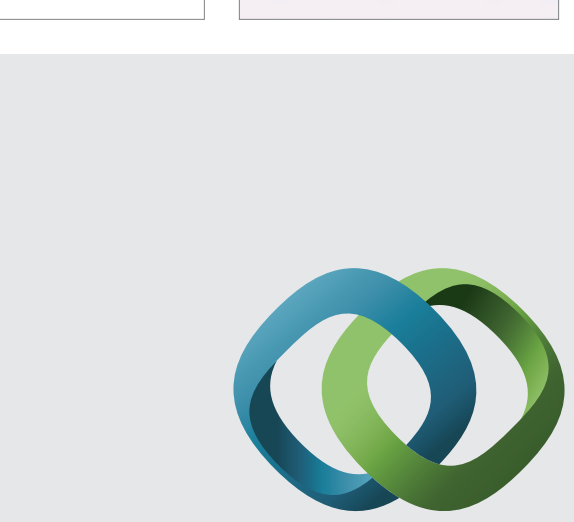

\section{Hindawi}

Submit your manuscripts at

http://www.hindawi.com
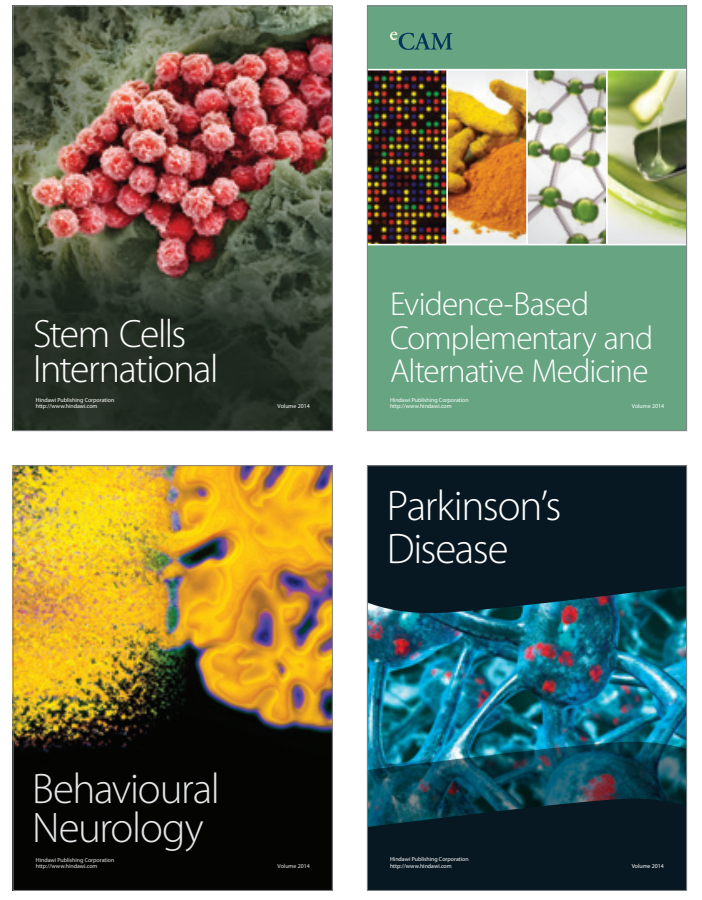
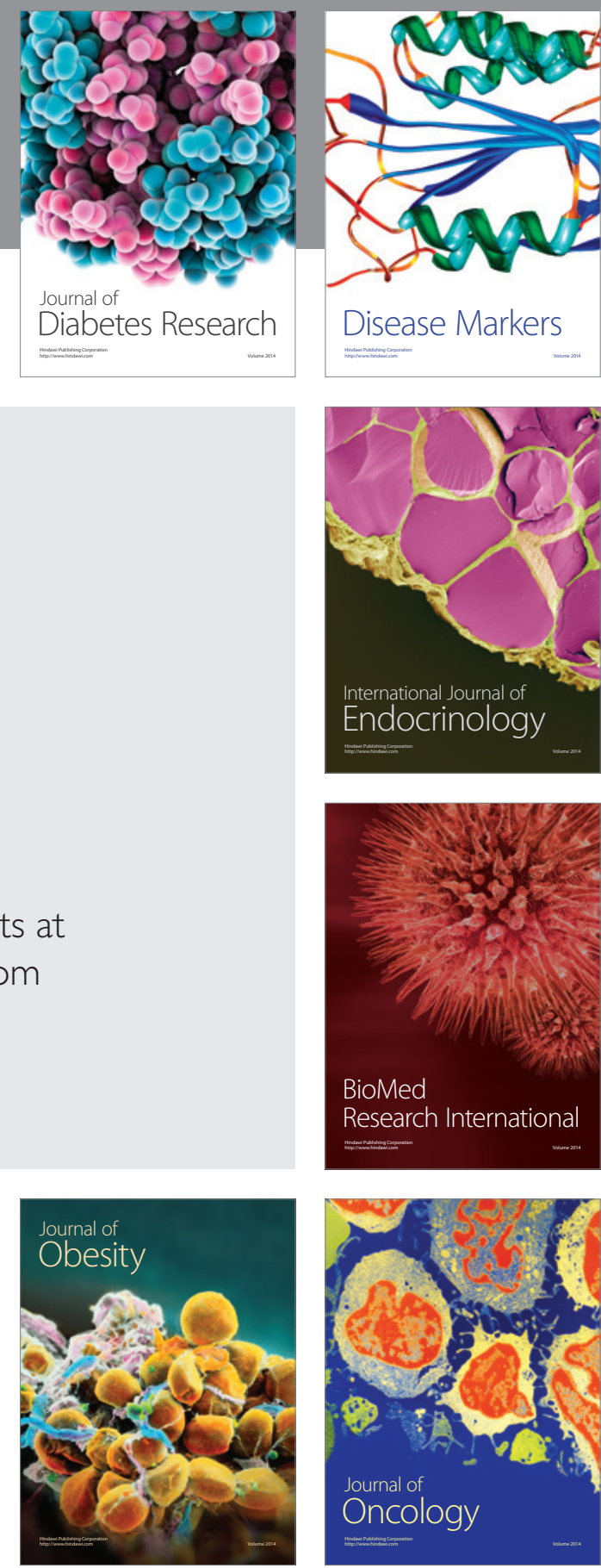

Disease Markers
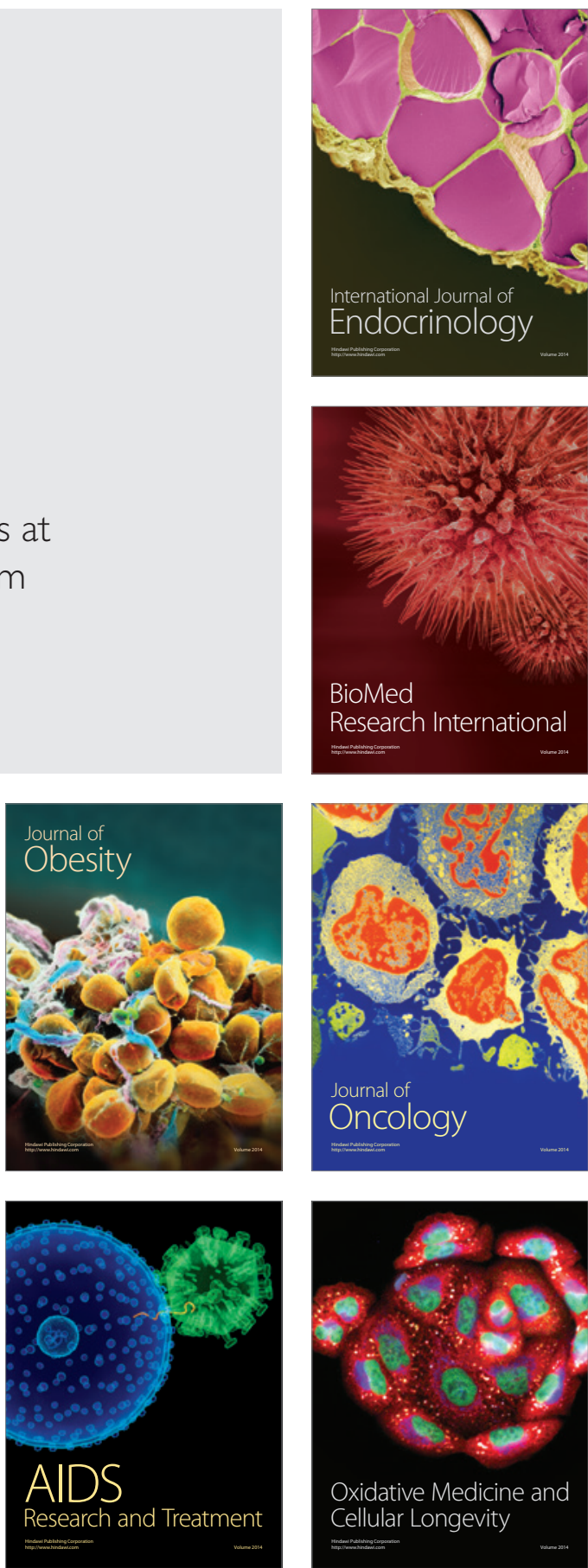